
allemande

50-2 | 2018

Humanités environnementales - Quoi de neuf du côté des méthodes?

\title{
Klimavulnerabilität von Unternehmen in der Metropolregion Oberrhein und ihre Visualisierung anhand von Wirkpfaden
}

Nicolas Scholze, Rüdiger Glaser et Sophie Roy

\section{(2) OpenEdition}

Édition électronique

URL : https://journals.openedition.org/allemagne/922

DOI : 10.4000/allemagne.922

ISSN : 2605-7913

Éditeur

Société d'études allemandes

Édition imprimée

Date de publication : 30 décembre 2018

Pagination : 325-335

ISSN : 0035-0974

Référence électronique

Nicolas Scholze, Rüdiger Glaser und Sophie Roy, „Klimavulnerabilität von Unternehmen in der Metropolregion Oberrhein und ihre Visualisierung anhand von Wirkpfaden ", Revue d'Allemagne et des pays de langue allemande [Online], 50-2 | 2018, Online erschienen am: 30 Dezember 2019, abgerufen am 19 Mai 2021. URL: http://journals.openedition.org/allemagne/922 ; DOI: https://doi.org/10.4000/ allemagne.922 


\title{
Klimavulnerabilität von Unternehmen in der Metropolregion Oberrhein und ihre Visualisierung anhand von Wirkpfaden
}

\author{
- Nicolas Scholze*, Rüdiger Glaser**, Sophie Roy***
}

\section{Einleitung und Hintergrund}

Der vorliegende Beitrag behandelt die Klimavulnerabilität von kleinen und mittleren Unternehmen (KMU) in der Trinationalen Metropolregion Oberrhein (TMO) und deren Visualisierung anhand von Wirkpfaden (auch Wirkungsketten oder engl. impact chains genannt). In diesem Visualisierungsverfahren werden branchenweise die von verschiedenen klimatischen Stressoren ausgehenden Kausalketten und deren Auswirkungen auf einzelne Unternehmensbereiche syntheseartig spezifiziert. Auch eine Abschätzung möglicher ökonomischer Folgen ist in den Wirkpfaden enthalten. Damit können sie einen Ansatzpunkt für zielgerichtete Anpassungsmaßnahmen bei betroffenen Unternehmen darstellen. Die Arbeiten wurden im Rahmen des InterregV-Forschungsvorhabens „Clim’Ability“ durchgeführt, an dem 13 Institutionen aus Deutschland, Frankreich und der Schweiz beteiligt waren.

Die TMO weist eine im mitteleuropäischen Vergleich hohe und komplexe Klimavulnerabilität auf ${ }^{(1)}$. Zum einen ist sie von einer global gesehen überdurchschnittlich starken Erwärmung betroffen ${ }^{(2)}$, die sich gemäß den aktuellsten Klimaprognosen auch

* Wissenschaftlicher Angestellter und Doktorand am Lehrstuhl von Prof. Glaser.

** Professor und Lehrstuhlinhaber der Physischen Geographie am Institut für Umweltsozialwissenschaften und Geographie der Albert-Ludwigs-Universität Freiburg i. Br.

*** Climatologue, ingénieur à Météo France.

1 Adelphi/PRC/EURAC, Vulnerabilität Deutschlands gegenüber dem Klimawandel, hg. vom Umweltbundesamt (Climate Change, 24/2015), Dessau-Roßlau, 2015, https://www.umweltbundesamt.de/sites/ default/files/medien/378/publikationen/climate_change_24_2015_vulnerabilitaet_deutschlands_ gegenueber_dem_klimawandel_1.pdf (04.07.2018).

2 Regionalverband Südlicher Oberrhein (Hg.), Regionale Klimaanalyse Oberrhein (REKLISO), bearbeitet von Prof. Dr. E. Parlow (Universität Basel), Prof. Dr. D. Scherer und Prof. Dr. U. Feh- 
in den nächsten Jahrzehnten fortsetzen wird ${ }^{(3)}$. Zum anderen führt die Erwärmung im Zusammenspiel mit der regionalen Topographie zu einer Zunahme bzw. Intensivierung von Extremereignissen wie Hitzewellen, Hochwasser, Starkregen oder Hagelunwettern. Auch mit einer Zunahme der Dürrewahrscheinlichkeit sowie einer Abnahme der Schneedeckendauer ist zu rechnen.

Die Unternehmen der Oberrheinregion werden in unterschiedlicher Art und Weise von den erwähnten klimatischen Veränderungen betroffen sein. Zudem fällt die Klimavulnerabilität auch innerhalb der TMO je nach Höhenlage und lokaler Topographie sehr unterschiedlich aus ${ }^{(4)}$.

Doch obwohl der Klimawandel seit Jahren in den Medien präsent ist und zu seiner Eindämmung bereits eine Vielzahl von Maßnahmen auf den verschiedensten Ebenen eingeleitet wurde, wird er in seinen lokalen Auswirkungen häufig nach wie vor unterschätzt. Individuelle Betroffenheit wird oft nicht wahrgenommen, was vor allem auf das fehlende Wissen um konkrete Folgen im lokalen Kontext zurückzuführen ist. In mehreren Studien wurde bei Unternehmen der Oberrheinregion ein Informationsbedarf in Bezug auf regional differenzierte Auswirkungen, Risiken und Chancen des Klimawandels festgestellt ${ }^{(5)}$. Um das Bewusstsein der eigenen Betroffenheit zu steigern, benötigen viele Unternehmen demnach zusätzliches Wissen über präzise benannte Auswirkungen des Klimawandels in ihrer Region und auf ihre Branche. Nur die detaillierte Kenntnis der Auswirkungen des Klimawandels auf den täglichen Betrieb ermöglicht es den Unternehmen, maßgeschneiderte Anpassungsmaßnahmen zu entwickeln und eventuell sich bietende Chancen frühzeitig zu erkennen.

\section{Konzeptionelle Aspekte von Klimavulnerabilität, Risiko und Wirkpfaden}

Die Analyse von Klimavulnerabilität ist Teil des breiter gefassten Risikodiskurses mit einer entsprechend vielfältigen theoretischen und konzeptionellen Hinterlegung $^{(6)}$. In der Risikoforschung wird Risiko üblicherweise als Funktion von Gefahr

renbach (beide TU Berlin), https://www.rvso.de/de/veroeffentlichungen/REKLISO_Inhalt_Daten sammlung.php (04.07.2018).

3 DWD (Deutscher Wetterdienst), Datensatz zu Klimaprojektionen eines Modellensembles für die Oberrheinregion (für das Projekt Clim'Ability zur Verfügung gestellt), 2017, unveröffentlicht.

4 Das kann aus verschiedenen Publikationen zur Klimavulnerabilität Deutschlands und Europas abgeleitet werden, z.B. EEA (European Environmental Agency), Climate change, impacts and vulnerability in Europe 2016. An indicator-based report, 2017, Luxembourg, https://www.eea.europa.eu/ publications/climate-change-impacts-and-vulnerability-2016 (04.07.2018); oder auch ADELPHI/PRC/ EURAC, Vulnerabilität Deutschlands gegenüber dem Klimawandel (Anm. 1).

5 Z.B. Projet SECIF „Vers des services climatiques pour les industries françaises“ (projet de recherche), Rapport final, 2015; oder Ministerium Für Umwelt, Klima und Energiewirtschaft Baden-Württemberg (Hg.), Strategie zur Anpassung an den Klimawandel in Baden-Württemberg, Stuttgart, https://um.baden-wuerttemberg.de/fileadmin/redaktion/m-um/intern/Dateien/ Dokumente/4_Klima/Klimawandel/Anpassungsstrategie.pdf (04.07.2018).

6 Ausführlich zum theoretischen Hintergrund bei J. Birkmann (Hg.), Measuring Vulnerability to Natural Hazards: Towards Disaster Resilient Societies, New York, 2013 (2. Aufl.). Zur Entwicklung der Vulnerabilitätskonzepte bei Hans-Martin Füssel \& Richard J.T. KLEIN, „Climate Change Vulnerability Assessments: An Evolution of Conceptual Thinking“, Climatic Change, 75/3 (2006), S. 301-329; H.G. Bohle \& T. Glade, „Vulnerabilitätskonzepte in Sozial- und Naturwissenschaften“, in: C. FeLGentreff \& T. Glade (Hg.), Naturrisiken und Sozialkatastrophen, Heidelberg, Elsevier/Spektrum 
(Hazard) und Verletzlichkeit (Vulnerabilität) definiert ${ }^{(7)}$. Um ein Risiko bewerten zu können, muss demnach sowohl die Frage nach allen potentiell möglichen Gefahren als auch nach der Vulnerabilität der den Gefahren ausgesetzten Elemente beantwortet werden, was in Gleichung (1) ausgedrückt ist:

(1) Risiko = Hazard x Vulnerabilität

Vulnerabilität wiederum wird wie in Gleichung (2) beschrieben als Funktion von Exposition, Sensitivität und Resilienz der untersuchten Elemente ${ }^{(8)}$. Während unter Exposition die Elemente verstanden werden, die im Eintrittsfall eines Ereignisses der Gefahr tatsächlich ausgesetzt sind, bezeichnet Sensitivität diejenigen der exponierten Elemente, welche empfindlich gegenüber der Gefahr sind. Mit Resilienz ist schließlich die Widerstandskraft der betroffenen Elemente gemeint, die häufig um das Konzept der Anpassungsfähigkeit ergänzt wird.

(2) Vulnerabilität $=($ Exposition $x$ Sensitivität $)-$ Resilienz

Der Begriff „Impact“ kann aus den beiden ersten Gleichungen abgeleitet werden. Er meint die Summe aller potentiell möglichen negativen Auswirkungen einer Gefahr und ist eine Funktion der Gefahr(en) selbst, der Exposition und der Sensitivität. Er unterscheidet sich vom Begriff „Risiko“ durch das Nicht-Berücksichtigen der Resilienz bzw. Anpassungsfähigkeit (adaptive capacity), die im Vulnerabilitätsbegriff mitgedacht wird und kann wie folgt definiert werden ${ }^{(9)}$ :

(3) Impact $=$ Hazard $\mathrm{x}$ (Exposition $\mathrm{x}$ Sensitivität $)$

Die Darstellung des Impacts in Form von Wirkpfaden ist eine v.a. in jüngeren Publikationen der angewandten Vulnerabilitäts- und Risikoforschung häufig verwendete, graphische Methode zur Visualisierung potentieller Negativwirkungen des Klimawandels auf die untersuchten Elemente. In einer groß angelegten Studie des Umweltbundesamtes zur Vulnerabilität Deutschlands gegenüber dem Klimawandel ${ }^{(10)}$ werden sie etwa benutzt, um Klimawirkungen in verschiedenen Handlungsfeldern wie Boden, Landwirtschaft, Fischerei, etc. darzustellen. Sie stellen in gewisser Weise einen operationalisierten Visualisierungsansatz gängiger Vulnerabilitätskonzepte dar.

Ausgangspunkt eines Wirkpfades ist stets ein Klimasignal, d.h. die prognostizierte Änderung eines klimatischen Parameters (Temperatur, Niederschlag, etc.). Die Auswirkungen des Signals werden nach verschiedenen Bereichen ausdifferenziert

Akademischer Verlag, 2007, S. 99-119; IPCC (Intergovernmental Panel on Climate Change), Climate Change 2014: Impacts, Adaption, and Vulnerability, Cambridge/New York, Cambridge University Press, 2014.

7 Z.B. Robert Chambers, „Editorial Introduction: Vulnerability, Coping and Policy“, IDS Bulletin, 20/2 (1989), S. 1-7, oder: Piers Blaikie, Terry Cannon, Ian Davis \& Ben Wisner, At Risk: Natural Hazards, People's Vulnerability, and Disasters, London, Routledge, 1994.

8 Nach Füssel/KLein, „Climate Change Vulnerability Assessments“ (Anm. 6).

9 Bundesministerium für wirtschaftliche Zusammenarbeit und Entwicklung (Hg.), The Vulnerability Sourcebook. Concept and guidelines for standardised vulnerability assessments, Bonn/ Eschborn, Deutsche Gesellschaft für Internationale Zusammenarbeit (GIZ) GmbH.

10 Adelphi/PRC/EURAC, Vulnerabilität Deutschlands gegenüber dem Klimawandel (Anm. 1). 
dargestellt, auch Interaktionen zwischen einzelnen Teilbereichen können abgebildet werden. Grundidee ist dabei, die dem Beziehungsgeflecht Klima-Gesellschaft bzw. Klima-Unternehmen innewohnende Komplexität und Vielschichtigkeit zu strukturieren und wesentliche Kausalitäten und Interaktionen sichtbar zu machen.

\section{Analyse der Klimavulnerabilität}

Im Rahmen des Forschungsvorhabens Clim'Ability wurden bislang 80 Interviews mit Unternehmen der TMO geführt, wobei die Interviewpartner sowohl aus deutschen als auch französischen und schweizerischen Unternehmen kamen. Im Interviewkorpus sind verschiedene Branchen vertreten, Schwerpunkte liegen in der Logistik, der Landwirtschaft und Nahrungsmittelindustrie, dem Tourismus, der Metallverarbeitung sowie der Energiewirtschaft.

Die Interviews wurden leitfadengestützt durchgeführt, wobei der Leitfaden branchenspezifisch angepasst wurde. Daneben wurde den Interviewpartnern eine standardisierte Checkliste vorgelegt, bei welcher der Impakt von klimatischen Stressoren auf einzelne Bereiche innerhalb des Unternehmens systematisiert abgefragt wurde. Diese Checkliste bildet eine wichtige Grundlage für die Kodierung und die Ableitung der Wirkpfade. Nach der Transkription wurden alle Interviews kodiert und hermeneutisch interpretiert.

Da Interview-Transkriptionen wie alle Texte mehrere Interpretationen zulassen, erwies sich die hermeneutische Interpretation ${ }^{(11)}$ als wichtiges Element bei der Interviewauswertung und damit auch bei der Ableitung der Wirkpfade. Die für hermeneutische Ansätze typische Systematik bestand darin, ein Transkript von mehreren Auswertern voneinander unabhängig kodieren zu lassen und anschließend die Zuordnungen zu einzelnen Kategorien (Stressoren, exponierte Bereiche, Folgen) in gemeinsamen Diskussionsrunden zu vergleichen. Dabei wurden zahlreiche Unterschiede in der Zuweisung von Codes auf Textstellen festgestellt, verschiedene Interpretationsmöglichkeiten herausgearbeitet und diskutiert sowie auf eine gemeinsame Auslegung hingearbeitet.

Die im Kontext von Clim'Ability abgeleiteten Wirkpfade orientieren sich zunächst an den Kategorien des Vulnerabilitätskonzepts, insbesondere an der oben genannten Definition von „Impact“. Die sich daraus ergebenden Kategorien „Hazard“, „Exposition“ und „Sensitivität“ wurden um eine Kategorie „mögliche Folgen und Risiken“ ergänzt, um die für Unternehmen relevanten ökonomischen Konsequenzen in die Visualisierung zu integrieren bzw. anzudeuten. Der Begriff „Hazard“ wurde durch den weiter gefassten Begriff „klimatische Stressoren“ ersetzt, um auch nicht mit einer akuten Gefahr assoziierte Klimawirkungen in die Analyse zu integrieren.

Insgesamt wurden im Laufe der Auswertung 11 klimatische Stressoren und 10 exponierte Bereiche definiert (s. Tab.1). Bei der Sensitivität hingegen wurden keine Kategorien vorab definiert oder während des Evaluierens festgelegt. Vielmehr wurden hier die in den Interviews beschriebenen, bereits aufgetretenen konkreten Probleme

11 Siehe z.B. Hans-Georg Gadamer, Wahrheit und Methode: Grundzüge einer philosophischen Hermeneutik, Tübingen, 1960 oder Annika Mattissek, Carmella Pfaffenbach \& Paul Reuber, Methoden der empirischen Humangeographie, Westermann, Braunschweig, 2013 (2. überarb. Aufl.) 
zusammengefasst. Die Liste der möglichen Sensitivitäten bleibt somit offen, was den Verfassern auf Grund der Vielschichtigkeit der unternehmerischen Tätigkeiten in der TMO als sinnvolle Vorgehensweise erschien. Dennoch konnten wiederholt auftretende Probleme zusammengefasst werden, z.B. das in zahlreichen Unternehmen virulente Problem des erhöhten Kühlenergiebedarfs bei einer zunehmenden Hitzebelastung.

Tab. 1: Klimatische Stressoren und exponierte Unternehmensbereiche im Rahmen von Clim'Ability

\begin{tabular}{|l|l|}
\hline Klimatische Stressoren & Exponierte Unternehmensbereiche \\
\hline Dürre & Personal \\
\hline Hitze & Prozesse \& Produktion \\
\hline Luftfeuchte & Management \\
\hline Starkregen & Kritische Infrastrukturen \\
\hline Hochwasser & Betriebsmittel \\
\hline Hagel & Lieferkette \\
\hline Gewitter mit Blitzschlag & Auftragslage \\
\hline Starkwind & Unternehmen (als Ganzes) \\
\hline Kälte / Eis & Rohstoffe \\
\hline Schnee & Sonstige \\
\hline Zunahme der mittleren Temperatur & \\
\hline
\end{tabular}

\section{Ergebnisse}

Im Folgenden sollen nun ausgewählte branchenspezifische und zugleich repräsentative Beispiele der im Rahmen von Clim'Ability erstellten Wirkpfade vorgestellt und diskutiert werden. Zu beachten ist, dass in den vorliegenden Beispielen aus Platzgründen nur eine Auswahl der für die jeweilige Branche abgeleiteten Wirkpfade dargestellt ist. Meist wurden diejenigen klimatischen Stressoren ausgewählt, die von den Interviewpartnern als die schwerwiegendsten eingestuft wurden. Dies bedeutet aber nicht, dass andere klimatische Stressoren keine Auswirkungen auf die untersuchten Branchen haben. Für eine vollständige Klimavulnerabilitätsanalyse müssten alle relevanten Stressoren untersucht und die daraus hervorgehenden Wirkpfade visualisiert werden.

\section{Straßenlogistik (Abb. 1)}

Im ersten Beispiel zur Straßenlogistik wurden die klimatischen Stressoren „Hitze“ und „Luftfeuchte“ als besonders relevant identifiziert und im weiteren Verlauf analysiert. Die Besonderheit des befragten Unternehmens ruht neben der klassischen Straßenlogistik in seiner umfassenden Kühllagerhaltung. Entsprechend wirken sich die identifizierten klimatischen Stressoren auf mehrere Bereiche aus, einige sind von beiden Stressoren betroffen. Besonders interessant ist der Zielkonflikt mit dem Brandschutz, der aufzeigt, dass der Klimawandel auch beim Gesetzgeber für Handlungsbedarf sorgt. Die möglichen Folgen und Risiken sind ebenfalls komplex und zeigen, wie grundlegend diese am analysierten Standort sind. 
Abb. 1: Wirkpfade in der Straßenlogistik

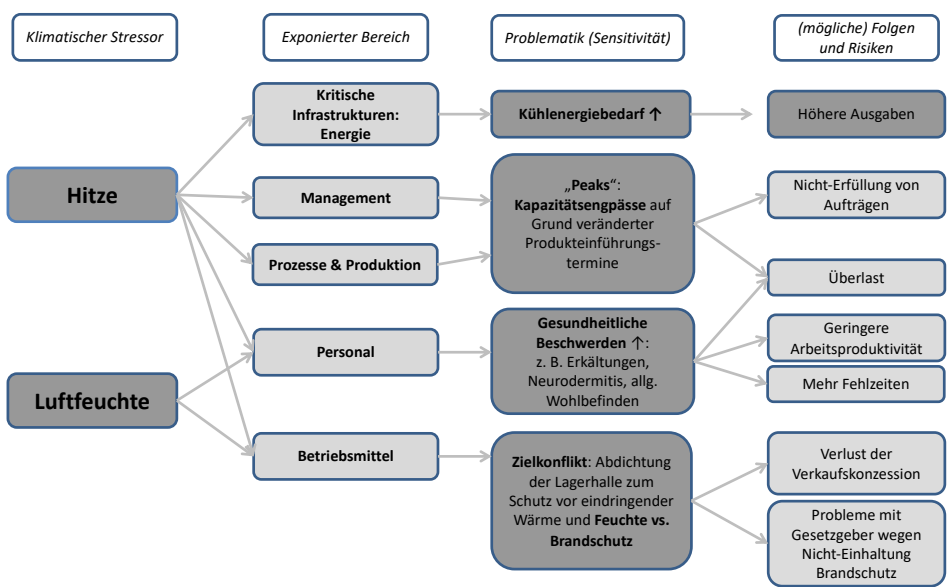

mögliche Folgen und Risiken: Kräftiges grau = im Interview erwähnt schwaches grau: Interpretation

Abb. 2: Wirkpfade in der Schifffahrtslogistik

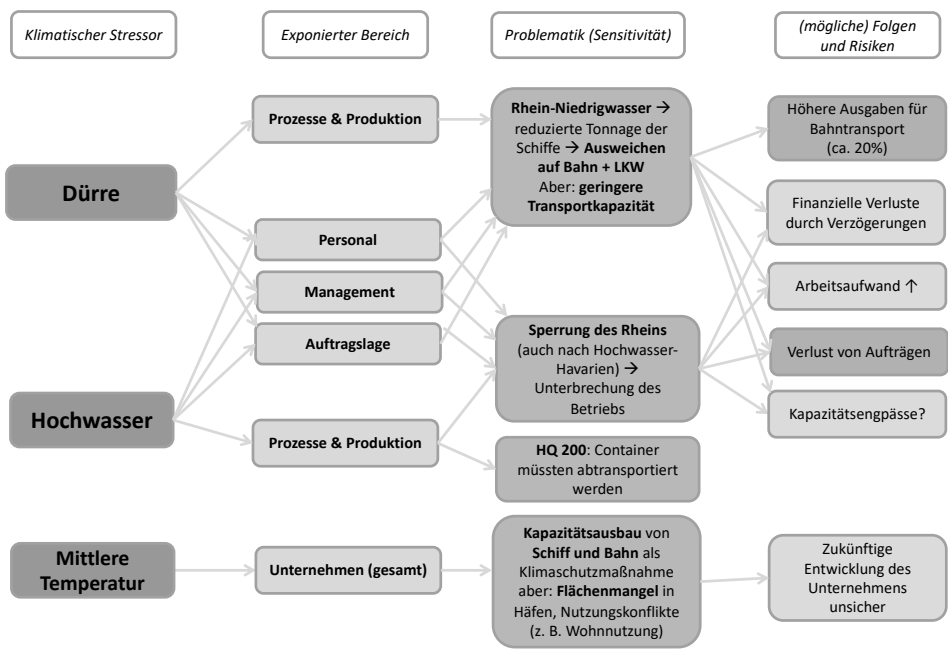

mögliche Folgen und Risiken: Kräftiges grau = im Interview erwähnt schwaches grau: Interpretation 


\section{Schiffahrtslogistik (Abb. 2)}

Die Klimavulnerabilität der Schifffahrtslogistik in der TMO unterscheidet sich sehr deutlich von derjenigen der Straßenlogistik, indem die Schifffahrt besonders von den gegensätzlichen Extremen Hoch- und Niedrigwasser in Folge von Dürren stark betroffen ist. Beide klimatischen Stressoren wirken sich gleichermaßen auf zentrale Unternehmensbereiche aus. Die Problematiken sind in der Ursache grundverschieden, im Ergebnis aber beinahe gleich, was sich in dem Überschneidungsmuster der Pfeile und den darin zum Ausdruck gebrachten Kausalitäten ausdrückt. Auch die Folgen und Risiken der beiden Stressoren ähneln sich stark und sind gleichermaßen negativ einzuschätzen. Der Anstieg der mittleren Temperatur ist davon abgesetzt und stellt weniger einzelne Bereiche als das gesamt Unternehmen mittelfristig vor größere Herausforderungen.

\section{Energieanbieter (Abb. 3)}

Die analysierten Energieanbieter erweisen sich ebenfalls als klimavulnerabel. Hier stehen die drei klimatischen Stressoren Dürre, Hitze und mittlere Temperaturen im Vordergrund. Im Unterschied zur Logistik wirken sie sich weniger auf einzelne Bereiche sondern vielmehr auf das Geschäftsmodell und damit auf das Unternehmen als Ganzes aus. Bemerkenswert sind die gegensätzlichen Auswirkungen in der Sensitivität und bei den möglichen Folgen und Risiken. Die analysierten Stressoren können sich sowohl gewinnsteigernd als auch ertragsmindernd auswirken. Derartige Befunde belegen die im Zuge der aktuellen Energiewende eher noch zunehmende Komplexität der Energiebranche und unterstreichen die Notwendigkeit individueller Analysen am Standort im Stile eines Audits, auch um die sich ergebenden Chancen möglichst frühzeitig nutzen zu können.

Abb. 3: Wirkpfade im Energiesektor

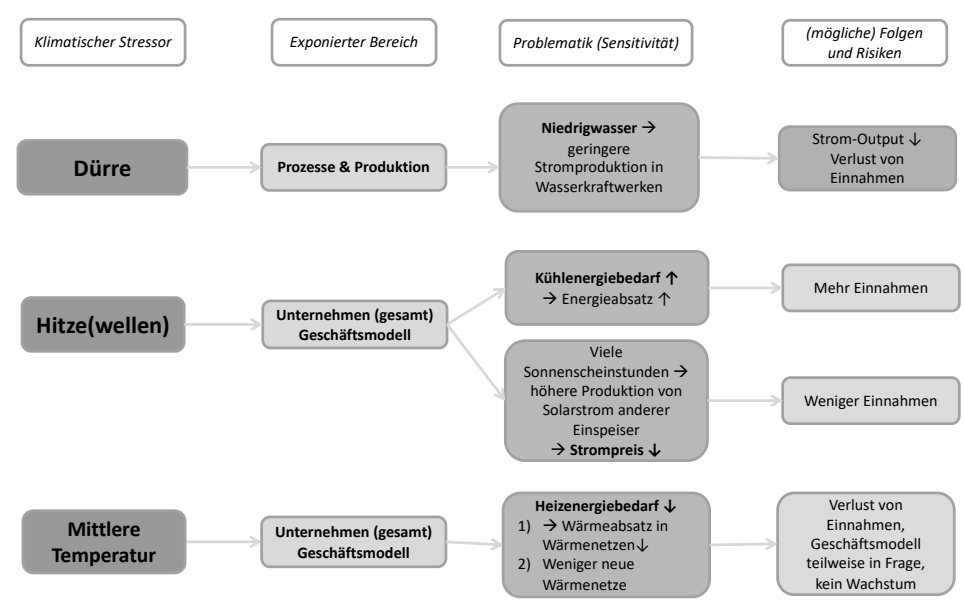

mögliche Folgen und Risiken: Kräftiges grau = im Interview erwähnt schwaches grau: Interpretation 


\section{Wintertourismus (Abb. 4)}

Der Wintertourismus in den Mittelgebirgen gilt als Paradebeispiel einer klimavulnerablen Branche ${ }^{(12)}$. Dies gilt auch für die höher gelegenen Regionen des südlichen Schwarzwaldes und der Vogesen, in denen der Wintertourismus eine hohe wirtschaftliche Bedeutung innehat. In der im Rahmen von Clim'Ability durchgeführten Fallstudie erweist er sich gegenüber dem Temperaturanstieg und dem damit einhergehenden Mangel an Schneesicherheit als besonders vulnerabel, aber auch gegenüber den Folgen von Stürmen, starkem Schneefall, extremer Kälte, Nebel und Regen. Sie führen in letzter Konsequenz über die ermittelte Sensitivität der betroffenen Bereiche zu durchweg negativen Folgen und damit einem hohen wirtschaftlichen Risiko. Insgesamt kann für diese Branche ein recht stringentes negatives Gesamtbild gezeichnet werden. Ergänzend ist aber hinzuzufügen, dass nicht alle unter „schlechtes Wetter“ subsummierten klimatischen Stressoren im Zuge des Klimawandels zunehmen werden, was besonders für extreme Kälte, aber auch für Winterstürme gilt. Zudem ist darauf hinzuweisen, dass der Sommertourismus in den untersuchten Gebieten vom aktuellen Klimawandel profitieren dürfte.

Abb. 4: Wirkpfade im Wintertourismus

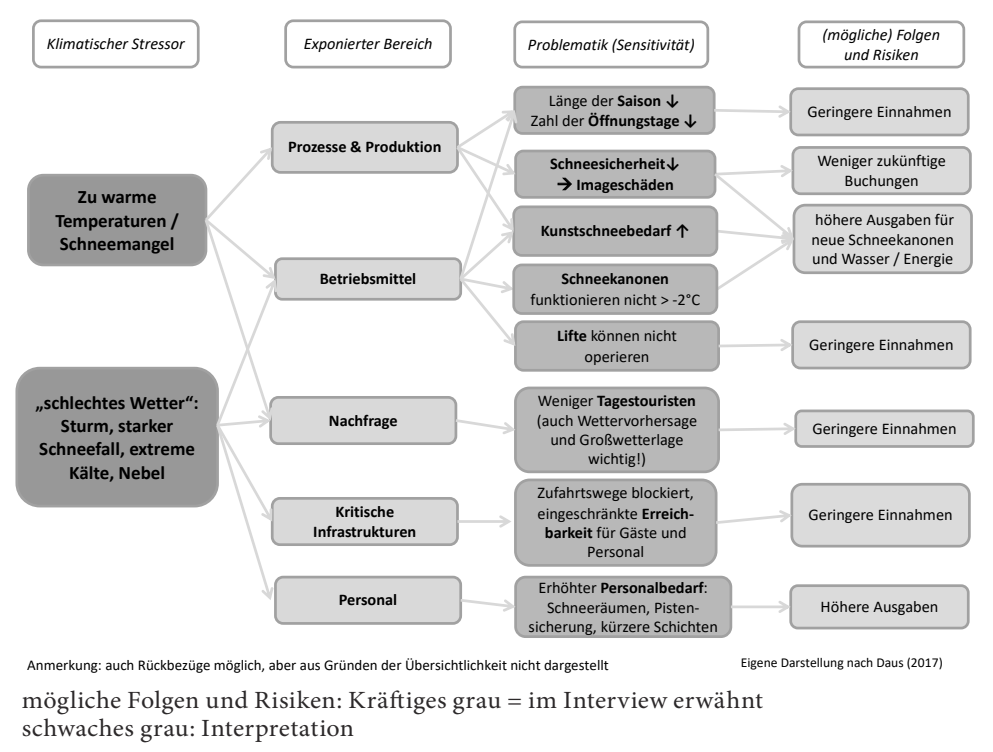

\section{Fazit}

Zunächst belegen die Analysen, dass Unternehmen und Branchen in der TMO vom Klimawandel und spezifischen klimatischen Stressoren ganz konkret am Standort betroffen sind. Dabei vermitteln die Wirkpfade in der dargestellten Form auf sehr schlüssige und kompakte Art und Weise, welche klimatischen Stressoren sich auf welche konkreten Bereiche in den einzelnen Unternehmen und Branchen auswirken. Sie 
verdeutlichen didaktisch einsichtig, inwiefern unternehmensinterne Prozessabläufe von externen klimatischen Stressoren beeinflusst werden und mit welchen Folgen und Risiken dies verbunden sein kann. In den Visualisierungen wird zudem durch unterschiedliche Farben markiert, ob die Risiken direkt im Gespräch erwähnt wurden, oder ob sich als logische Folge aus dem Gesagten ergeben. Sie sind somit zum Großteil auf die hermeneutische Interpretation der Interviews zurückzuführen. Von den befragten Unternehmen können sie als Hinweis auf mögliche Folgen gedeutet werden und als Ansatzpunkt für künftige Anpassungsmaßnahmen dienen.

Insgesamt sind die Folgen und Risiken oft vielschichtig und komplex, was ihre Identifizierung und Kommunikation schwierig gestaltet. Auch wird deutlich, dass verschiedene Branchen unterschiedlich stark betroffen sind, dass aber auch innerhalb der Branchen oft gegensätzliche Wertungen und Gewichtungen vorliegen, beispielweise innerhalb der Logistikbranche mit unterschiedlichen Einschätzungen zwischen Straßen- und Schifffahrtslogistik. Auch ist nicht jeder Stressor gleichermaßen für alle Branchen relevant.

Die branchenspezifischen klimatischen Stressoren und Auswirkungen lassen auch eine den klimatischen Charakteristika der einzelnen Teilbereiche der TMO entsprechende Prägung erkennen, d.h. die auf die Höhenstandorte bezogenen Branchen wie der Wintertourismus oder die in den von Hitze und Dürre stärker betroffenen Logistikunternehmen in den Tieflagen reflektieren in hohem Maße die regionalspezifischen klimatischen Besonderheiten.

\section{Diskussion und Ausblick}

Die durchgeführten Analysen belegen und verdeutlichen die branchenspezifische Klimavulnerabilität von Unternehmen in der TMO recht anschaulich. Die auf über 80 Interviews basierenden Auswertungen, die den Kriterien der empirischen Sozialforschung folgen und konzeptionell an Risikoansätzen orientiert sind, lassen sich über die besondere Visualisierungsform von Wirkpfaden didaktisch sinnvoll und kompakt umsetzen. Die in den Interviews identifizierten klimatischen Stressoren und deren Auswirkungen auf sensible Bereiche mit der weiteren Konkretisierung und Einschätzung der Folgen und Risiken werden dabei sichtbar und in generalisierter Form vermittelt. Die Visualisierungsform zeichnet sich zudem durch eine gewisse Dynamik aus, die über eher statische Darstellungen wie Balken- oder Radardiagramme hinausgeht und wenig robuste quantitative Elemente wie etwa das reine Auszählen von Nennungen eines klimatischen Stressors in den Interviews vermeidet. Trotz der dabei getroffenen Verknappung lassen sich komplexe Interaktionen ebenso abbilden wie zum Teil gegensätzliche Folgen und Wirkungen. Dies kann als Beleg für die Operationalisierbarkeit angesehen werden ${ }^{(13)}$. In der Synopsis zeigen sich aber auch die branchenspezifischen Unterschiede und Gegensätze. Entsprechend positiv fiel auch die Rückmeldung von Pilotunternehmen aus, welche diese Visualisierungsform als einsichtig, stringent und damit positiv bewerteten.

13 Zur Operationalisierung von Klimavulnerabilität: R. Glaser, N. Scholze \& S. Jergentz, Klimavulnerabilität von Unternehmen im regionalen Kontext, Lehrbuch Geographie, Springer, 2018 (3. Aufl. in print). 
Andererseits bringt auch diese Methode einige Schwierigkeiten mit sich. So ist beispielsweise die Zuordnung der Auswirkungen besonders zu exponierten Bereichen nicht immer eindeutig. Oftmals sind mehrere Zuordnungen möglich, was wiederum die Abbildung komplexer und damit schwerer lesbar macht. Daneben ist im vorliegenden Fall die Anzahl der Interviews pro Branche noch zu gering, um statistisch valide Aussagen zu generieren. Die dargestellten Wirkpfade sind somit als rein qualitative Aussagen zu interpretieren, die noch einer statistischen Überprüfung mit ausreichend großer Stichprobe bedürften. Dies wiederum ist mit hohem Aufwand verbunden. Und schließlich bleibt die Interpretation der vorgestellten Beispiele trotz des hermeneutischen Ansatzes immer auch bis zu einem gewissen Grad subjektiv, von Vorwissen und Weltanschauung der Auswertenden sowie dem Zeitgeist und weiteren Faktoren beeinflusst, sodass die Möglichkeit anderer Interpretationen besteht. Ein Lösungsansatz wäre hier, den befragten Unternehmen die Evaluierung vorzulegen und um Rückmeldung bezüglich der Plausibilität der Wirkpfade zu bitten. Dieser Schritt wurde im Rahmen von Clim'Ability bislang nur mit einigen Pilotunternehmen umgesetzt, konnte aber in den entsprechenden Fällen die Qualität und Robustheit der Wirkpfade sicherlich erhöhen.

Selbstredend ersetzen die Wirkpfade in ihrer generalisierten Form nicht detailreichere, textbasierte Darstellungsformen. Um Klimavulnerabilität abschließend beurteilen zu können sind auch umfassendere Ansätze, in welchen die Resilienz der Unternehmen Beachtung findet, nötig. Nichtsdestotrotz können Wirkpfade aber als eine stimmige Visualisierungsform der Auswirkungen von Klimawandelstressoren auf Unternehmen angesehen werden, die einen raschen Überblick und einen Einstieg in die Thematik ermöglichen. Werden sie als Werkzeug im Rahmen von Klimavulnerabilitätsdiagnosen eingesetzt, besitzen sie auf Grund ihrer Übersichtlichkeit und den konkret benannten Sensitivitäten zudem das Potential, den Unternehmen präzise Hinweise auf bisher unentdeckte Schwachstellen oder mögliche Anpassungsmaßnahmen zu liefern.

\section{Zusammenfassung}

Die Unternehmen der Trinationalen Metropolregion Oberrhein (TMO) sind auf sehr unterschiedliche Art und Weise vom Klimawandel betroffen, wobei die relevanten klimatischen Stressoren wie Hitzewellen, Starkregen, Hochwassergefahr, etc. kleinräumig und branchenspezifisch stark differieren. Zudem fehlt es vielen, besonders kleineren und mittleren Unternehmen (KMU) an präzisen und anwendungsorientierten Informationen über die konkreten Auswirkungen des Klimawandels an ihrem Standort und auf ihre Branche, sodass sie oft keine genaue Vorstellung von ihrer tatsächlichen Klimavulnerabilität haben. Um diesem Informationsdefizit Rechnung zu tragen wurde im Rahmen des Forschungsprojektes Clim'Ability die Klimavulnerabilität einiger Branchen in der TMO analysiert und in Form von Wirkpfaden visualisiert. In diesem Verfahren werden branchenweise die von verschiedenen klimatischen Stressoren ausgehenden Kausalketten und deren Auswirkungen auf einzelne Unternehmensbereiche syntheseartig spezifiziert und um eine Abschätzung möglicher ökonomischer Folgen für das Unternehmen ergänzt. Damit können sie einen Ansatzpunkt für zielgerichtete 
Anpassungsmaßnahmen bei betroffenen Unternehmen darstellen. Besonders bei KMU ohne eigene Umweltabteilung sind die vorgestellten Wirkpfade somit als Beitrag zur Stärkung der Resilienz gegenüber negativen Klimafolgen denkbar.

\title{
Résumé
}

Les entreprises du Rhin supérieur sont affectées par le changement climatique de différentes manières. Les facteurs de stress climatiques pertinents, tels que les vagues de chaleur, les fortes pluies, les inondations, etc., varient selon la position géographique et la branche d'activité de l'entreprise. De plus, beaucoup d'entre elles, particulièrement les petites et moyennes entreprises (PME), manquent d'informations précises et utilisables sur les impacts du changement climatique sur leur localisation et leur secteur spécifique, de sorte qu'elles ont souvent une connaissance lacunaire de leur propre vulnérabilité climatique. Pour compenser ce déficit d'information, la vulnérabilité au climat de quelques secteurs d'activité abordés dans le cadre du projet de recherche Clim'Ability a été analysée et représentée par des chaînes d'impact. Ces chaînes d'impact décrivent la chaîne complète des causalités déclenchées par un facteur de stress climatique et synthétise son impact sur les domaines exposés dans l'entreprise, ainsi que les conséquences économiques possibles. Elles peuvent être un point de départ pour la mise en place de mesures d'adaptation ciblées pour les entreprises concernées. Les chaînes d'impact apportent des éléments destinés à renforcer la résilience des entreprises aux impacts négatifs du changement climatique, particulièrement pour des PME qui n'ont pas de service dédié aux questions environnementales.

\begin{abstract}
The enterprises in the Trinational Metropolitan Region Upper Rhine are affected by climate change in a multitude of ways. The relevant climatic stressors such as heatwaves, heavy rain, flood risk, etc. differ greatly on the local scale and sectorwise. In addition, many enterprises, particularly small and medium sized ones (SME), are often lacking precise and practical information on the impacts of climate change at their specific location and on their specific sector, which causes an often scarce knowledge of their own climate vulnerability. In order to meet this need of information and to highlight the results of climate vulnerability assessment of some sectors carried out within the Clim'Ability research project, impact chains were derived and visualized. The resulting impact chains comprise the whole chain of causalities triggered by a climatic stressor synthesizing its impacts on exposed domains within the enterprise as well as the possible economical consequences. By providing such information to the affected enterprises, the impact chains might be used as a starting point for practice-oriented adaptation measures. Especially for SME without environmental departments they can be discussed as a contribution to enhance their resilience against negative impacts of climate change.
\end{abstract}

